\title{
FAM3A enhances adipogenesis of 3T3-L1 preadipocytes via activation of ATP-P2 receptor-Akt signaling pathway
}

\author{
Yujing Chi ${ }^{1,}{ }^{*}$, Jing $\mathrm{Li}^{2}{ }^{2}{ }^{*}, \mathrm{Na} \mathrm{Li}^{1}$, Zhenzhen $\mathrm{Chen}^{3}$, Liping Ma ${ }^{1}$, Weikang Peng ${ }^{1}$, Xiuying \\ Pan ${ }^{1}$, Mei $\mathrm{Li}^{1}$, Weidong $\mathrm{Yu}^{1}$, Xiangjun $\mathrm{He}^{1}$, Bin Geng ${ }^{3}$, Qinghua Cui ${ }^{4}$, Yulan Liu ${ }^{1,2}$ \\ and Jichun Yang ${ }^{3}$ \\ ${ }^{1}$ Institute of Clinical Molecular Biology \& Central Laboratory, Peking University People's Hospital, Beijing 100044, China \\ ${ }^{2}$ Department of Gastroenterology, Peking University People's Hospital, Beijing 100044, China \\ ${ }^{3}$ Department of Physiology and Pathophysiology, School of Basic Medical Sciences, Peking University Health Science Center, \\ Key Laboratory of Cardiovascular Science of the Ministry of Education, Center for Non-coding RNA Medicine, Beijing 100191, \\ China \\ ${ }^{4}$ Department of Biomedical Informatics, School of Basic Medical Sciences, Peking University Health Science Center, Key \\ Laboratory of Cardiovascular Science of the Ministry of Education, Center for Non-coding RNA Medicine, Beijing 100191, \\ China \\ *These authors have contributed equally to this work
}

Correspondence to: Jichun Yang, email: yangj@bjmu.edu.cn Yulan Liu, email: liuyulan@pkuph.edu.cn

Keywords: FAM3A, PPAR $\gamma$, adipogenesis, ATP, P2 receptor

Received: June 07, $2016 \quad$ Accepted: April 23, 2017

Published: May 03, 2017

Copyright: Chi et al. This is an open-access article distributed under the terms of the Creative Commons Attribution License 3.0 (CC BY 3.0), which permits unrestricted use, distribution, and reproduction in any medium, provided the original author and source are credited.

\section{ABSTRACT}

FAM3A plays important roles in regulating hepatic glucose/lipid metabolism and the proliferation of VSMCs. This study determined the role and mechanism of FAM3A in the adipogenesis of 3T3-L1 preadipocytes. During the adipogenesis of 3T3-L1 preadipocytes, FAM3A expression was significantly increased. FAM3A overexpression enhanced 3T3-L1 preadipocyte adipogenesis with increased phosphorylated Akt (pAkt) level, whereas FAM3A silencing inhibited 3T3-L1 preadipocyte adipogenesis with reduced pAkt level. Moreover, FAM3A silencing reduced the expression and secretion of adipokines in 3T3L1 cells. FAM3A protein is mainly located in mitochondrial fraction of 3T3-L1 cells and mouse adipose tissue. FAM3A overexpression increased, whereas FAM3A silencing decreased ATP production in 3T3-L1 preadipocytes. FAM3A-induced adipogenesis of 3T3-L1 preadipocytes was blunted by inhibitor of P2 receptor. In white adipose tissues of $\mathrm{db} / \mathrm{db}$ and HFD-fed obese mice, FAM3A expression was reduced. One-month rosiglitazone administration upregulated FAM3A expression, and increased cellular ATP content and pAkt level in white adipose tissues of normal and obese mice. In conclusion, FAM3A enhances the adipogenesis of preadipocytes by activating ATP-P2 receptor-Akt pathway. Under obese condition, a decrease in FAM3A expression in adipose tissues plays important roles in the development of adipose dysfunction and type 2 diabetes.

\section{INTRODUCTION}

Obesity, a clinical condition characterized by the excessive accumulation of body fat, is a strong risk factor for type 2 diabetes $[1,2]$. PPAR $\gamma$ is one of the key transcription factors that regulate and promote adipogenesis, and its agonists such as rosiglitazone and pioglitazone exerted significant hypoglycemic effects [3].
However, one significant side effect of PPAR $\gamma$ agonists is bodyweight gain in both clinical studies and animal experiments $[4,5]$. Transgenic mice with adipose-specific overexpression of PPAR $\gamma$ display obesity and increased global insulin sensitivity, and are protected against high fat diet (HFD)-induced insulin resistance and hyperglycemia [6]. In support, specific deletion of PPAR $\gamma$ in adipose causes lipodystrophy, global insulin resistance and 
hyperglycemia in mice [7]. This paradox of obesity and type 2 diabetes suggested that more studies are still needed to explore the role of adipose tissues in the development of type 2 diabetes under obese condition. Adipose dysfunction is also called adiposopathy or sick fat, which is mainly characterized by increased lipolysis and disturbed adipokine expression profile. Obese patients with adipose dysfunction are at higher risk of type 2 diabetes than those without adipose dysfunction [8-16]. When chronic exposure to excessive nutrients, expansion and adipogenesis of preadipocytes are necessary for storing lipid or glucose as TG $[11,14,17]$. Under the condition of excessive nutrients, an impairment in the capacity of preadipocyte adipogenesis will cause adipose dysfunction, leading to ectopic lipid deposition and disturbed adipokine expression/secretion [8, 18].

The family with sequence similarity 3 (FAM3) gene family is a cytokine-like gene family consists of four members designated FAM3A, FAM3B, FAM3C, and FAM3D, respectively. FAM3A is a target gene of PPAR $\gamma$, and activation of PPAR $\gamma$ induced FAM3A expression in liver cells [19]. In the livers of obese diabetic mice and patients with non-alcoholic fatty liver disease (NAFLD), FAM3A expression was reduced. Hepatic activation of FAM3A markedly attenuated hyperglycemia, insulin resistance and fatty liver in obese diabetic mice via the activation of Akt signaling pathway [20]. So far, whether FAM3A regulates the adipogenesis of preadipocytes remains unknown.
A

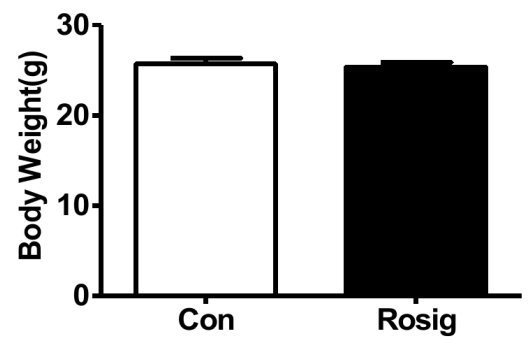

B

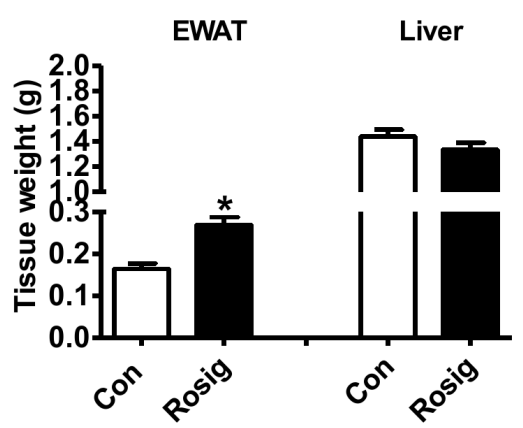

C

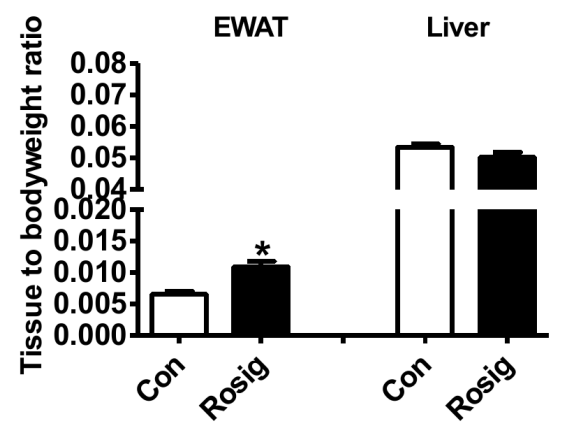

D

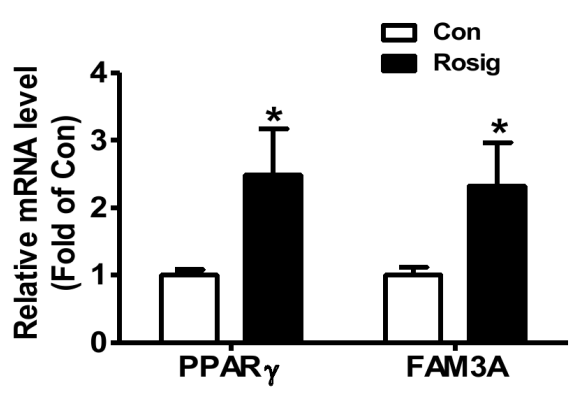

E

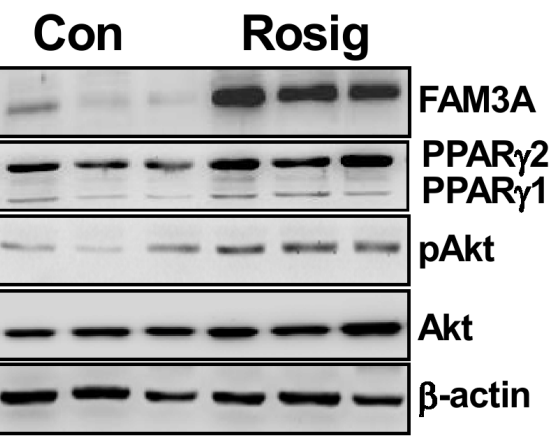

F

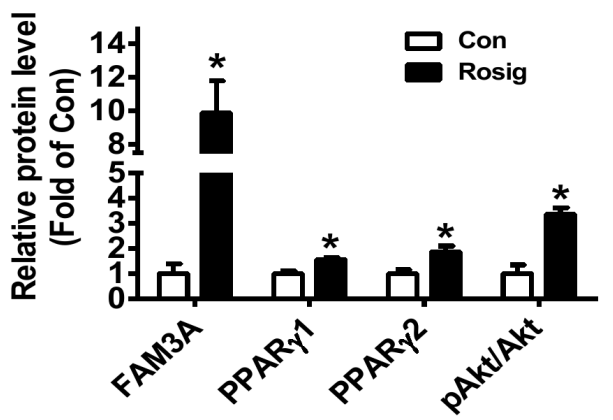

Figure 1: Rosiglitazone administration upregulated FAM3A expression in adipose tissues of C57BL/6 mice. 8-10 week old C57BL/6 mice fed on normal diet were orally administrated with rosiglitazone for 1 month. (A-C) Rosiglitazone administration on bodyweight (A), epididymal white adipose tissue (EWAT) and liver weight (B), and tissue weight/bodyweight ratios (C). (D) Rosiglitazone administration on the mRNA levels of PPAR $\gamma$ and FAM3A in adipose tissues. The primer for PPAR $\gamma$ mRA analysis detected both PPAR $\gamma 1$ and PPAR 2 mRNA in adipose tissues in this study. (E, F) Rosiglitazone administration on the protein levels of PPAR $\gamma$ and FAM3A in adipose tissues. Representative gel images were shown in (E), and quantitative data shown in (F). Con: control mice treated with saline; Rosig: experiment mice treated with rosiglitazone. $\mathrm{N}=6-8$ for protein assays, $\mathrm{N}=12-14$ for $\mathrm{mRNA}$ assays, ${ }^{*} \mathrm{p}<0.05$ versus control mice. 
In the current study, we reported that FAM3A enhances the adipogenesis of 3T3-L1 preadipocytes by activating ATP-P2 receptor-Akt signaling pathway. In white adipose tissues of obese diabetic mice, FAM3A expression was reduced. Rosiglitazone administration upregulated FAM3A expression in HFD-fed diabetic mouse tissues with improved adipokine profile and hyperglycemia. These findings suggested that a decrease in FAM3A expression in adipose tissues contributes to the development of adipose dysfunction and type 2 diabetes.

\section{RESULTS}

\section{Rosiglitazone administration upregulated FAM3A expression in epididymal white adipose tissue of C57BL/6 mice}

To initially determine whether FAM3A is involved in adipogenic process, C57BL/6 mice were orally administrated with rosiglitazone for one month, and then FAM3A expression in white adipose tissue was determined. Although rosiglitazone treatment failed to affect whole bodyweight (Figure 1A), it increased the weight of epididymal white adipose tissue (EWAT) (Figure 1B, 1C). The mRNA and protein levels of PPAR $\gamma$ and FAM3A were increased by rosiglitazone treatment in EWAT of mice (Figure 1D, 1E). Moreover, the phosphorylated Akt (pAkt) level was also increased by rosiglitazone treatment in EWAT of mice (Figure 1E, 1F).

\section{FAM3A expression was increased during the adipogenesis of 3T3-L1 preadipocytes}

To further confirm that FAM3A is involved in adipogenic process, its expression in differentiated 3T3$\mathrm{L} 1$ preadipocytes was determined. When compared to the normally differentiated 3T3-L1 cells, Oil Red O staining revealed that rosiglitazone induced more neutral lipid deposition on differentiation Day 4 and Day 8 (Figure 2A). In normally differentiated 3T3-L1 cells, the mRNA levels of PPAR $\gamma$ and FAM3A were increased on Day 4 and Day 8 (Figure 2B, 2C) when compared with that on Day 0. Moreover, rosiglitazone induced further elevation in PPAR $\gamma$ and FAM3A mRNA levels when compared with normally differentiated cells (Figure 2B). The protein levels of PPAR $\gamma 2$ and FAM3A were increased

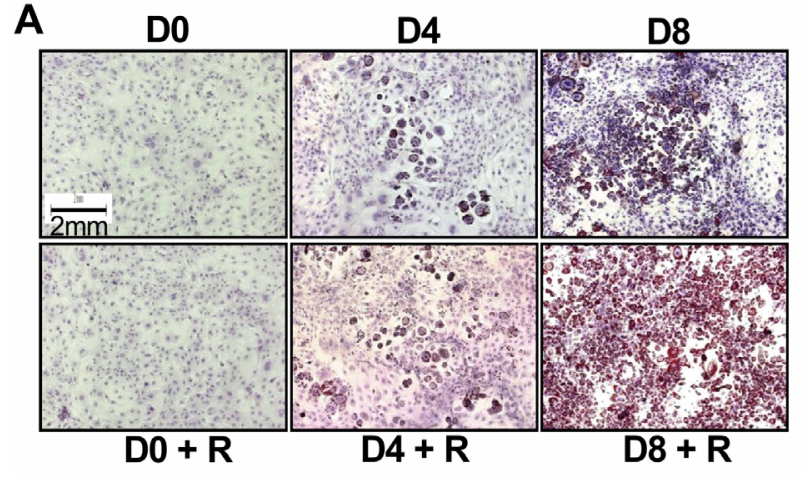

B
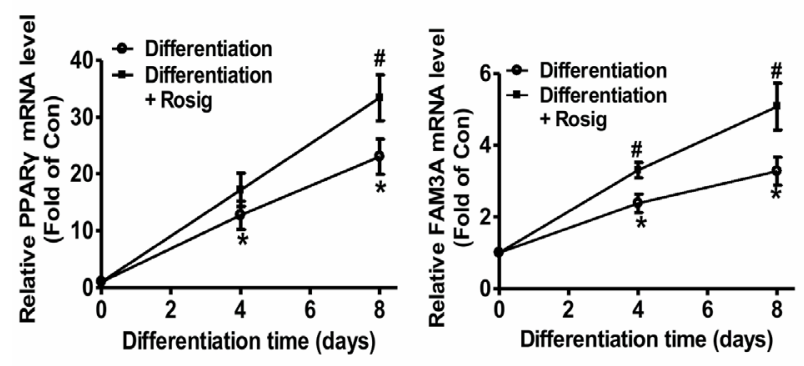

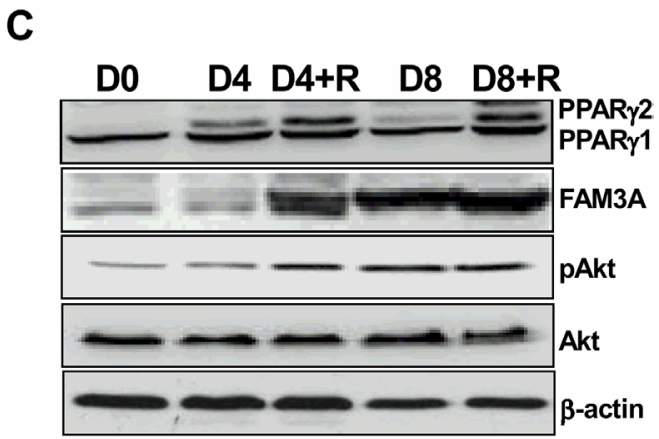

D

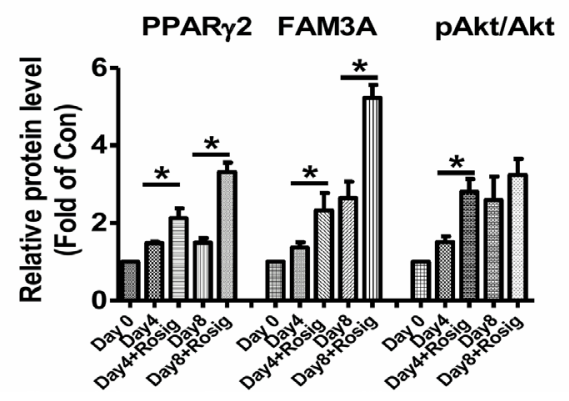

Figure 2: FAM3A expression was increased in differentiated 3T3-L1 preadipocytes. (A) Representative images of differentiated 3T3-L1 cells with Oil Red O staining. The magnification power is 100. (B) The mRNA levels of PPAR $\gamma$ and FAM3A during differentiation of 3T3-L1 cells. The primer for PPAR $\gamma$ mRNA analysis detected both PPAR $\gamma 1$ and PPAR $\gamma 2$ mRNA in 3T3-L1 cells in this study. (C, D) The protein levels of PPAR $\gamma$ and FAM3A during differentiation of 3T3-L1 cells. Representative gel images were shown in (C), and quantitative data shown in (D). D0, D4, and D8 represented 0 day, 4 days and 8 days after induction of differentiation, respectively; $\mathrm{R}$, in the presence of rosiglitazone. $\mathrm{N}=5-8,{ }^{*} \mathrm{p}<0.05$ versus $\mathrm{D} 0$ or control, $\# \mathrm{p}<0.05$ versus differentiation group without rosiglitazone at the corresponding time point. 
(Figure 2C, 2D), whereas that of PPAR $\gamma 1$ remained unchanged during adipogenesis of 3T3-L1 cells (Figure 2C, 2D). pAkt level was increased on Day 4 and Day 8 when compared with Day 0. Rosiglitazone induced further elevation in PPAR $\gamma 2$ and FAM3A protein levels on Day 4 and Day 8, but only induced further increase in pAkt level on Day 4 but not Day 8 (Figure 2C, 2D). The mRNA levels of adipokines including aP2, CEBP $/ \alpha$, $\mathrm{CEBP} / \delta$, adiponectin and resistin were elevated during the adipogenesis of 3T3-L1 cells (Supplementary Figure $1)$. The mRNA levels of aP2, $\mathrm{CEBP} / \alpha$, adiponectin and resistin were further induced by rosiglitazone in 3T3-L1 cells (Supplementary Figure 1).

\section{FAM3A silencing inhibited the differentiation of 3T3-L1 cells}

To directly evaluate the impact of FAM3A on the adipogenesis of 3T3-L1 preadipocytes, its expression was knockdown using siRNA interference. FAM3A mRNA level was reduced about $67 \%$ in 3 T3-L1 preadipocytes after siFAM3A treatment for 24 hours, and remained lower on Day 4 and Day 8 when compared with control cells (Figure 3A). FAM3A knockdown reduced the mRNA level of PPAR $\gamma$ on Day 4 and Day 8 (Figure 3A).
siFAM3A treatment significantly reduced the protein levels of FAM3A, PPAR $\gamma 2$ and pAkt on Day 0, Day 4 and Day 8 when compared with control cells. Oil Red $\mathrm{O}$ staining and quantitative assays revealed that FAM3A silencing significantly reduced neutral lipid deposition and adipogenesis of 3T3-L1 cells on Day 4 and Day 8 (Figure 3C, 3D). FAM3A silencing reduced the mRNA levels of adipokine $\mathrm{aP} 2, \mathrm{C} / \mathrm{EBP} \alpha$, adiponectin and resistin, whereas it had little effect on that of $\mathrm{C} / \mathrm{EBP} \delta$ in $3 \mathrm{~T} 3-\mathrm{L} 1$ cells on Day 8 (Supplementary Figure 2A-2E). FAM3A silencing reduced adiponectin secretion on Day 4, whereas it reduced resistin secretion on Day 4 and Day 8 in 3T3-L1 cells (Supplementary Figure 2F, 2G).

\section{FAM3A overexpression enhanced the differentiation of 3T3-L1 cells}

FAM3A was overexpressed in 3T3-L1 cells to further confirm its adipogenic role in preadipocyte adipogenesis. On Day 0, Day 4 and Day 8 post viral infection, Ad-FAM3A-transduced cells exhibited significantly higher FAM3A mRNA level when compared with Ad-GFP-treated cells. FAM3A overexpression had no significant effect on the mRNA level of PPAR $\gamma$ (Figure 4A). Ad-FAM3A treatment significantly increased

\section{A}

C
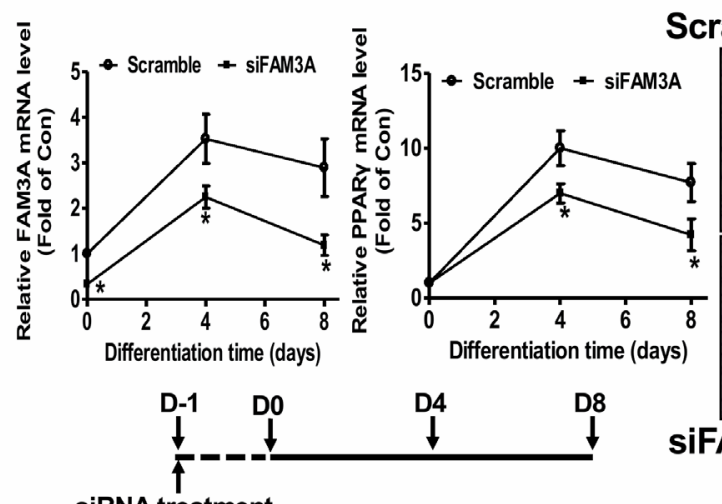

SIFAM3A DO

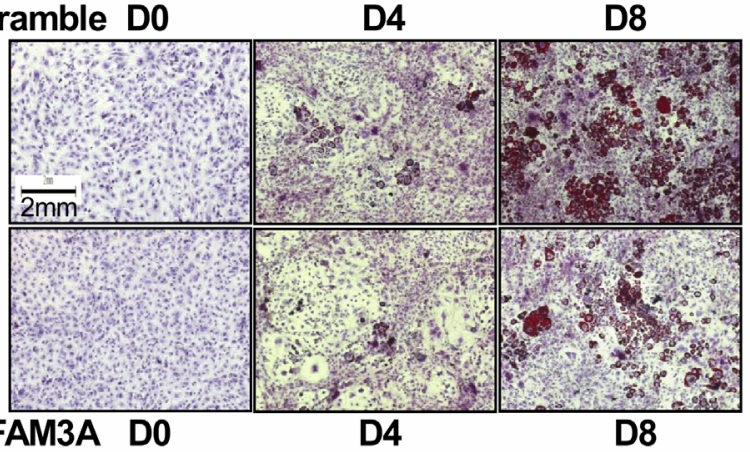

siRNA treatment

B

D0 D4 D8

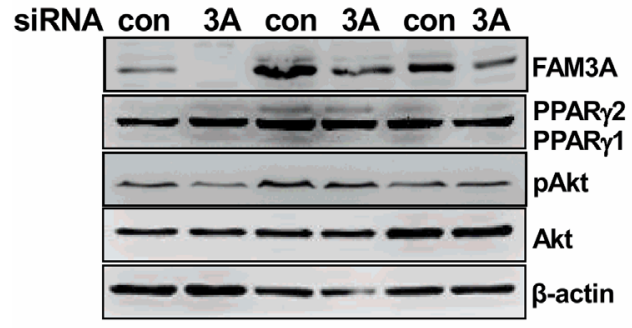

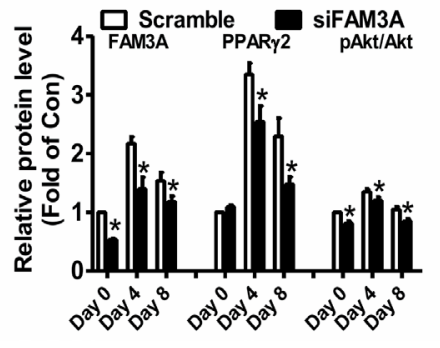

Figure 3: siRNA-mediated silencing of FAM3A inhibited the differentiation of 3T3-L1 preadipocytes. (A) siFAM3A treatment on the mRNA levels of FAM3A and PPAR $\gamma$ in 3T3-L1 cells. (B) siFAM3A treatment on the protein levels of FAM3A and PPAR $\gamma$ in 3T3-L1 cells. Representative gel images were shown in left panel, and quantitative data shown in right panel. (C) Representative images of differentiated 3T3-L1 cells with Oil Red O staining after treatment with siFAM3A or scrambled siRNA. (D) Quantitative analysis of TG levels in differentiated 3T3-L1 cells. siFAM3A: cells treated with siFAM3A; scramble: cells treated with scrambled siRNA. N=6, ${ }^{*}$ p $<0.05$ versus control groups treated with scrambled siRNA at the corresponding time point. 
FAM3A protein level on Day 0, Day 4 and Day 8, and PPAR $\gamma 2$ protein level on Day 4 and Day 8 when compared with control cells. Moreover, FAM3A overexpression increased pAkt level on Day 0 and Day 8 (Figure 4B). Oil Red $\mathrm{O}$ staining and quantitative assays revealed that FAM3A overexpression enhanced neural lipid deposition and adipogenesis of in 3T3-L1 preadipocytes (Figure 4C, 4D). FAM3A overexpression failed to further increase the mRNA levels of adipokines (Supplementary Figure $3 \mathrm{~A}-3 \mathrm{E}$ ), and the secretion of adiponectin and resistin in 3T3-L1 preadipocytes (Supplementary Figure 3F, 3G).

\section{FAM3A enhanced the adipogenesis of 3T3-L1 preadipocytes via ATP-P2 receptor-Akt signaling pathway}

Our previous study had revealed that FAM $3 \mathrm{~A}$ is a novel mitochondrial protein [20, 21]. FAM3A protein is also predominantly located in mitochondrial fraction of mouse adipose tissue and 3T3-L1 cells (Supplementary Figure 4A, 4B). In proliferated 3T3-L1 cells, extracellular ATP content was increased (Figure 5A). FAM3A silencing reduced extracellular ATP content on Day 0 and Day 8
(Figure 5B), whereas FAM3A overexpression elevated extracellular ATP content on all time points (Figure 5C). One-month rosiglitazone treatment upregulated FAM3A expression and increased cellular ATP content in adipose tissues of C57BL/6 mice (Figure 5D). To determine whether FAM3A enhances the adipogenesis of 3T3-L1 cells via ATP signaling pathway, P2 receptor (ATP receptor) signaling was blocked using PPADS. Because there is no significant adipogenesis on Day 0, the experiment of Day 0 had not been performed here. In Ad-GFP-treated cells, adipogenesis was not significantly affected by PPADS treatment on Day 4 and Day 8 (Figure 5E, 5F). In contrast, PPADS treatment significantly repressed FAM3A-stimulated adipogenesis on Day 4 and Day 8 (Figure 5E, 5F). In support, FAM3A-induced increase in pAkt and PPAR $\gamma 2$ protein levels was blunted by PPADS treatment (Figure 5G, 5H). Although PPADS treatment failed to affect PPAR $\gamma 2$ protein level and adipogenesis in Ad-GFP-infected cells, it reduced pAkt level on Day 4 and Day 8 (Figure 5G, 5H). On Day 4 and Day 8, PPADS treatment reduced PPAR $\gamma$ mRNA level in Ad-FAM3A-treated cells but not in Ad-GFP-treated cells (Supplementary Figure 5A). PPADS treatment reduced the mRNA levels of aP2 and resistin on Day 8 in Ad-FAM3Atreated cells (Supplementary Figure 5B-5F).

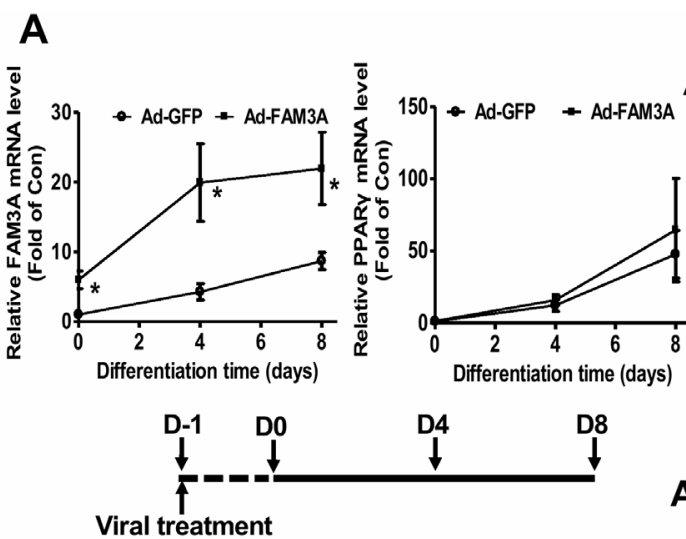

C

Ad-GFP DO

D4

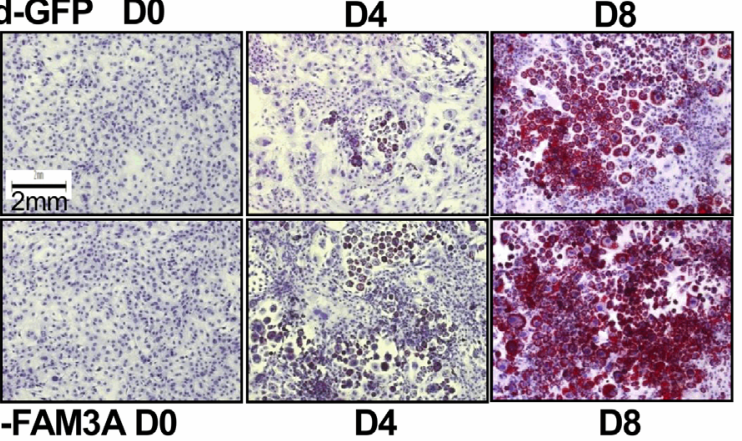

\section{B}

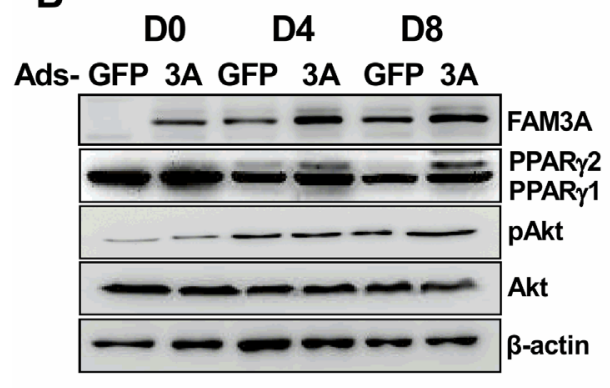

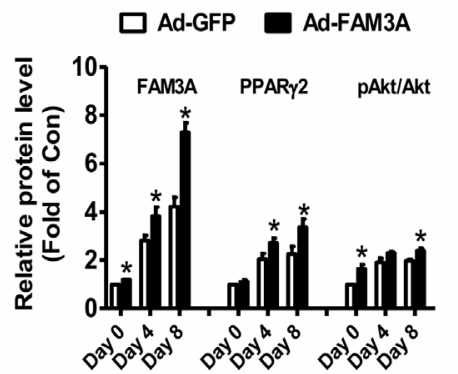

D

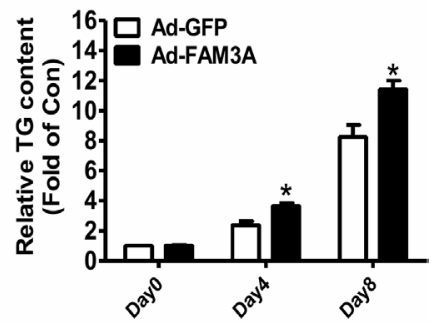

Figure 4: FAM3A overexpression enhanced the differentiation of 3T3-L1 preadipocytes. (A) Ad-FAM3A treatment on the mRNA levels of FAM3A and PPAR $\gamma$ in 3T3-L1 cells. (B) Ad-FAM3A treatment on the protein levels of FAM3A and PPAR $\gamma$ in 3T3L1 cells. Representative gel images were shown in left panel, and quantitative data shown in right panel. (C) Representative images of differentiated 3T3-L1 cells with Oil Red O staining after Ad-FAM3A or Ad-GFP treatment. (D) Quantitative analysis of TG levels in differentiated 3T3-L1 cells. $\mathrm{N}=5-8,{ }^{*} \mathrm{p}<0.05$ versus control groups treated with Ad-GFP at the corresponding time point. 
FAM3A is reduced in adipose tissues of HFD mice and $\mathrm{db} / \mathrm{db}$ mice

The mRNA and protein levels of FAM3A and PPAR $\gamma$ were reduced in $\mathrm{db} / \mathrm{db}$ (Figure 6A, 6B) and HFD mouse white adipose tissues (Figure 6C, 6D). PPAR $\gamma$ overexpression significantly activated the promoter activity of mouse FAM3A gene in 3T3-L1 cells, further supporting that PPAR $\gamma$ directly activated FAM3A expression in adipocytes (Supplementary Figure 6). Rosiglitazone treatment increased PPAR $\gamma 2$ protein level but not PPAR $\gamma 1$ protein level in adipose tissue of HFDmice (Figure 7A, 7B). The mRNA level of FAM3A, and the protein levels of FAM3A and pAkt were increased in rosiglitazone-treated adipose tissue of HFD mice (Figure 7A-7C). The mRNA levels of aP2 and C/EBP $\delta$ were significantly increased, whereas that of resistin was decreased in rosiglitazone-treated adipose tissue (Figure 7A). The ATP content was also increased in HFD mouse adipose tissue by rosiglitazone treatment (Figure 7D).
siRNA-mediated knockdown of PPAR $\gamma$ failed to affect FAM3A-induced Akt phosphorylation at the beginning of 3T3-L1 preadipocyte differentiation (Figure 8A). Moreover, although PPAR $\gamma$ knockdown inhibited the differentiation of 3T3-L1 preadipocytes, FAM3A similarly promoted the differentiation of 3T3-L1 preadipocytes transfected with scrambled siRNA or siPPAR $\gamma$ (Figure 8B).

\section{DISCUSSION}

So far, the biological functions of FAM3A remain largely unknown. FAM3A is a target gene of PPAR $\gamma$ [19]. In this study, gain- and loss-function evaluation revealed that FAM3A enhanced preadipocyte adipogenesis. FAM3A activates Akt via ATP-P2 receptor pathway in liver cells and vascular smooth muscular cells [20,21]. There had been several lines of evidences suggesting that mitochondrial ATP production is associated with the adipogenesis of preadipocytes. Several key subunits of ATP synthase including ATP synthase alpha and beta

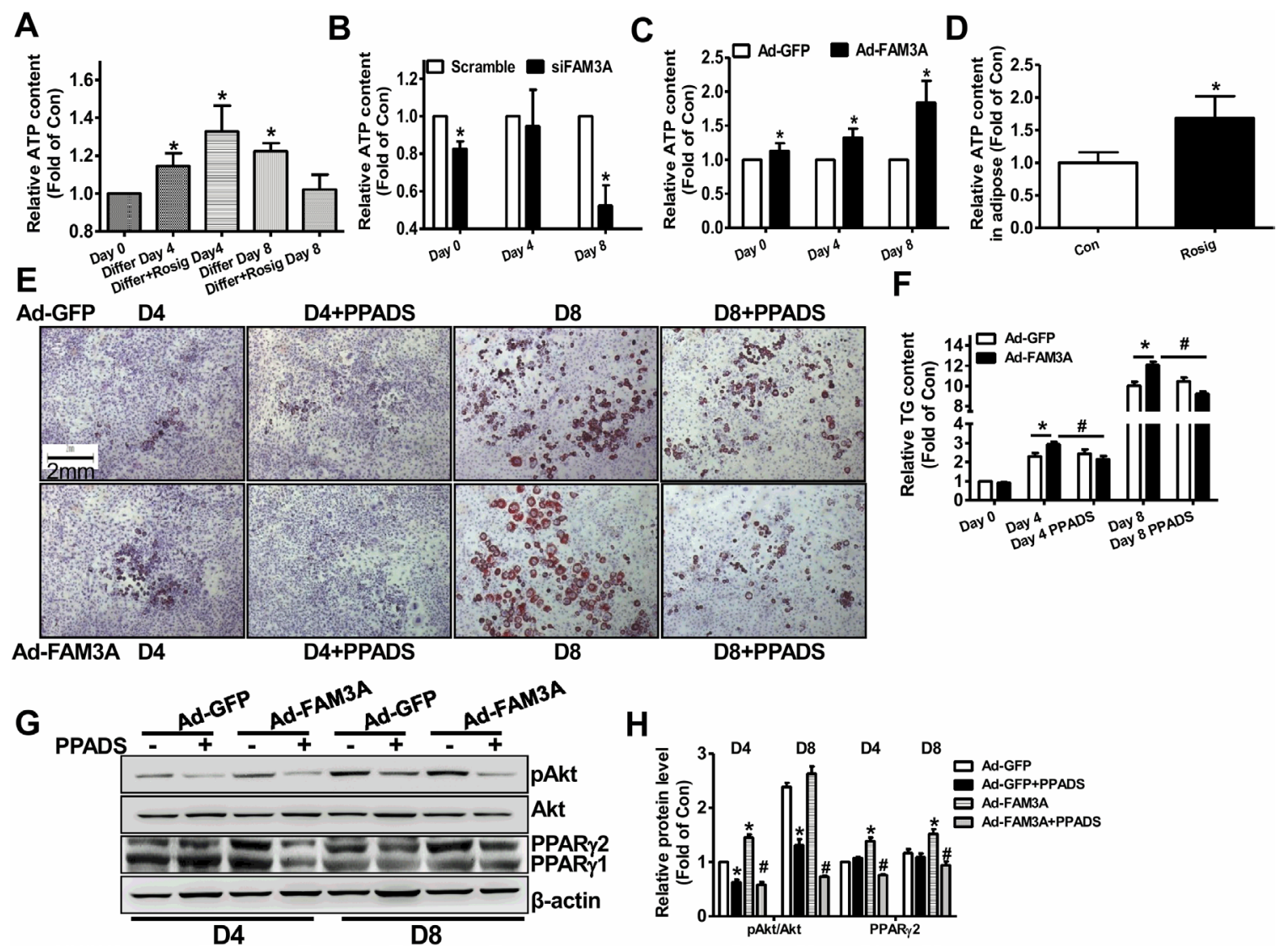

Figure 5: Inhibition of P2 receptor blocked FAM3A-induced adipogenesis of 3T3-L1 preadipocytes. (A-C) Extracellular ATP content in differentiated 3T3-L1 cells (A), siFAM3A-treated 3T3-L1 cells (B), and Ad-FAM3A-treated 3T3-L1 cells (C). ATP content in the medium of these cells were determined as described in experimental procedure. (D) ATP content in adipose tissues of C57BL/6 mice treated with rosiglitazone for 1 month. (E) Representative images of differentiated 3T3-L1 cells with Oil Red O staining after treatment with Ad-FAM3A or Ad-GFP in the absence or presence of PPADS. (F) Quantitative analysis of TG levels in differentiated 3T3-L1 cells in panel $(\mathbf{E})$. (G, H) Inhibition of P2 receptor blocked FAM3A-induced Akt activation in 3T3-L1 cells. Representative gel images were shown in $(\mathbf{G})$, and quantitative data shown in $\mathbf{( H )} . \mathrm{N}=6,{ }^{*} \mathrm{p}<0.05$ versus control groups treated with Ad-GFP at the corresponding time point; $\# \mathrm{p}<0.05$ versus Ad-FAM3A-treated group. 
A

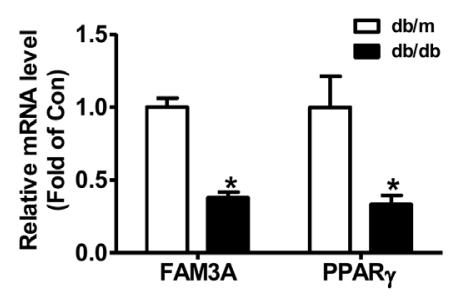

B

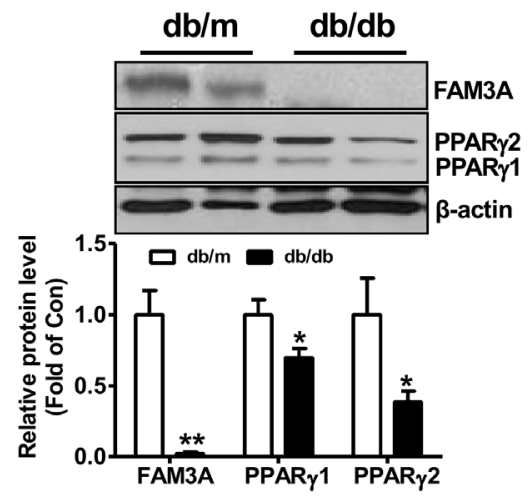

C

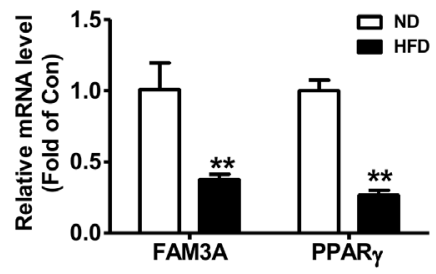

D

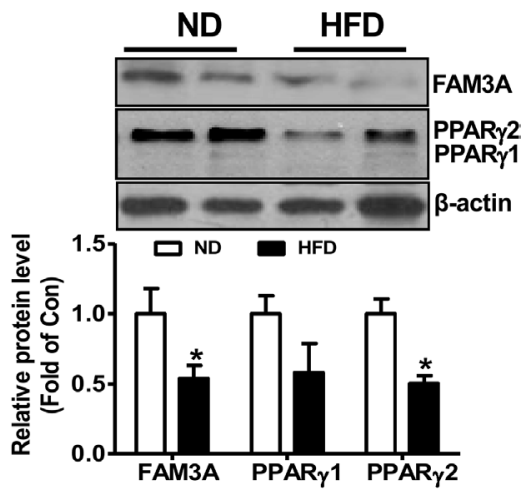

Figure 6: FAM3A expression was reduced in white adipose tissues of obese diabetic mice. (A, B) In adipose tissues of db/db mice, the mRNA (A) and protein (B) levels of FAM3A and PPAR $\gamma$ were reduced when compared with $\mathrm{db} / \mathrm{m}$ mice. (C, D) In adipose tissues of mice fed on HFD for 12 weeks, the mRNA (C) and protein (D) levels of FAM3A and PPAR $\gamma$ were reduced when compared with mice fed a normal chow. ND: normal diet; HFD: high fat diet. $\mathrm{N}=5-8,{ }^{*} \mathrm{p}<0.05$ versus control mice.

A

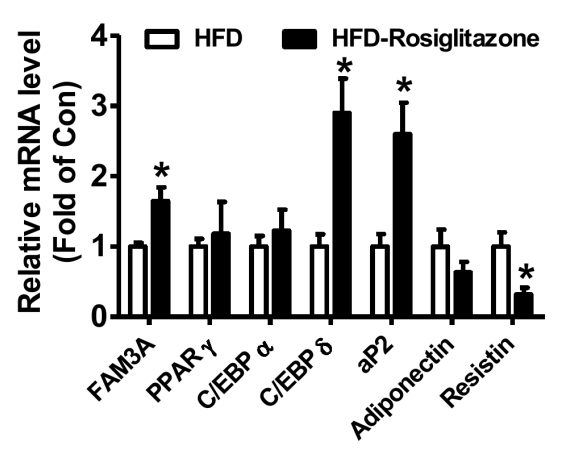

B

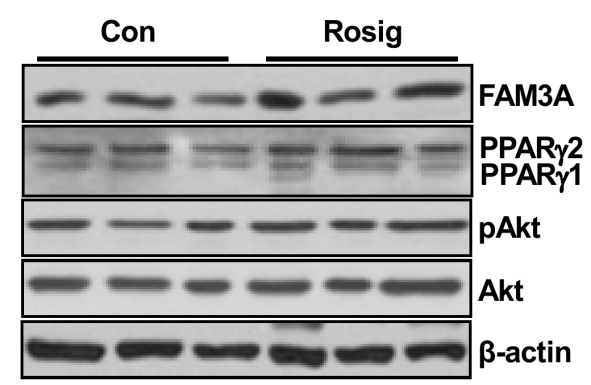

C

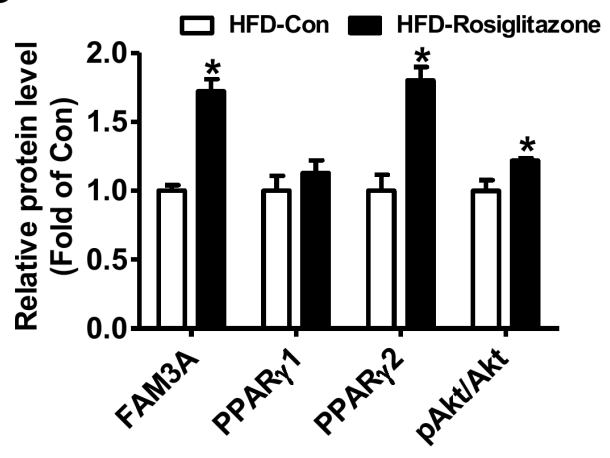

D

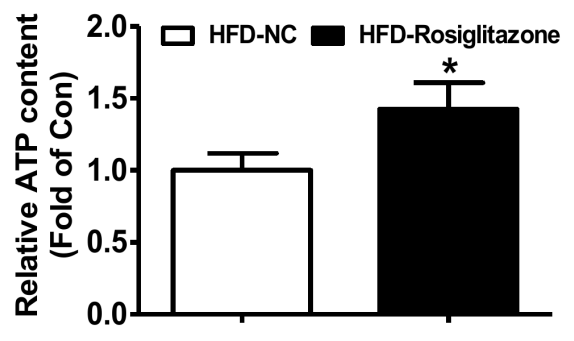

Figure 7: Rosiglitazone administration upregulated FAM3A expression in white adipose tissues of mice fed on HFD for 12 weeks. C57BL/6 mice were fed on a HFD or normal chow for 12 weeks, followed by oral treatment with rosiglitazone for 1 month. (A) Rosiglitazone treatment on the mRNA levels of FAM3A and adipokines in adipose tissues. $(\mathbf{B}, \mathbf{C})$ Rosiglitazone treatment on the protein levels of FAM3A, PPAR $\gamma$ and pAkt in adipose tissues. (D) Rosiglitazone treatment increased cellular ATP content in adipose tissues. N=8, ${ }^{*} \mathrm{p}<0.05$ versus control mice treated with saline. 
subunits were increased during adipogenesis of 3T3-L1 cells [22]. Although addition of exogenous ATP alone failed to stimulate adipogenesis of 3T3-L1 preadipocytes, it augmented the adipogenic effects of other hormones by activating P2Y receptors [23]. Depletion of mitoferrin 1/2 reduced mitochondrial ATP production and repressed the adipogenesis of 3T3-L1 preadipocytes [24]. In support, overexpression of uncoupling protein 2 (UCP-2) impaired ATP production and induced the apoptosis of 3T3-L1 cells [25]. In adipose tissues of obese mice, several key genes controlling ATP production were reduced, but reversed by rosiglitazone treatment with the improvement of insulin resistance and hyperglycemia [26]. Activation of Akt pathways has been reported to play important roles in promoting adipogenesis of 3T3-L1 cells in several studies [27-30]. However, the impact of endogenous ATP production and its signaling pathway(s) on adipogenesis of preadipocytes still remains unclear.

Our findings demonstrated that FAM3A protein is predominantly located in mitochondrial fraction in adipose tissues and 3T3-L1 cells. FAM3A overexpression increased, whereas FAM3A silencing decreased ATP production in 3T3-L1 cells. Moreover, rosiglitazone administration also upregulated FAM3A expression, and increased ATP production in adipose tissues in C57BL/6 mice. Finally, PPADS, an antagonist of P2 receptor [20], blocked FAM3A-induced Akt activation and adipogenesis of 3T3-L1 cells. Clearly, FAM3A stimulates the adipogenesis of preadipocytes by activating ATP-P2 receptor-Akt signaling pathway. A decrease in FAM3A expression in adipose tissues might be a novel mechanism for explaining adipose dysfunction under obese condition [8, 18]. Restoration of FAM3A expression in adipose tissue may represent potential therapeutical strategy for correcting adipose dysfunction. In Ad-GFPinfected 3T3-L1 cells, PPADS treatment also reduced Akt phosphorylation but failed to significantly affect adipogenesis. This suggested that ATP-P2 receptorAkt signaling axis is not the only pathway triggering adipogenesis in basal condition without FAM3A overexpression. In contrast, PPADS repressed FAM3Ainduced Akt activation and adipogenesis in 3T3-L1
A

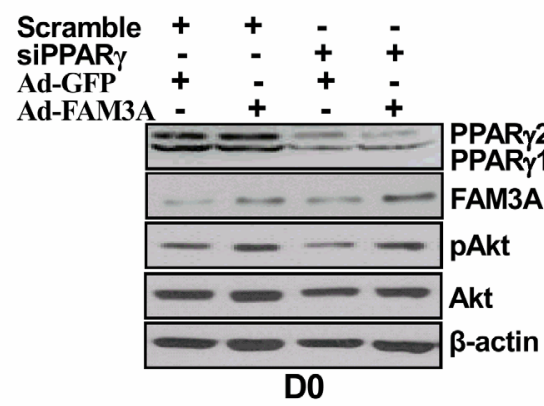

B

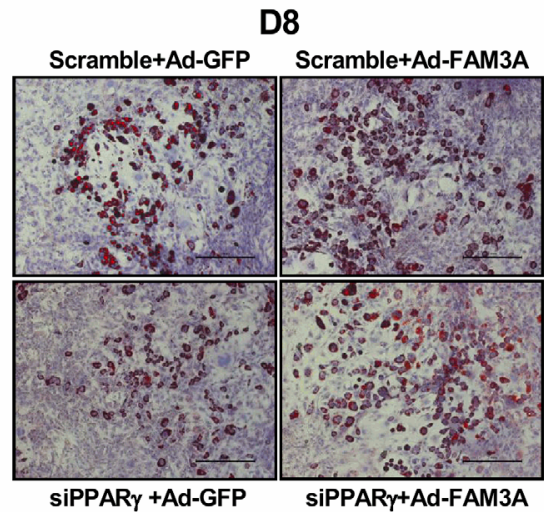

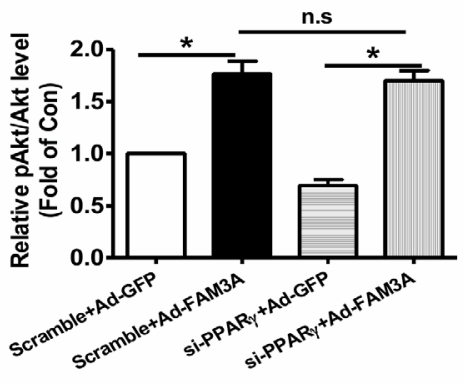

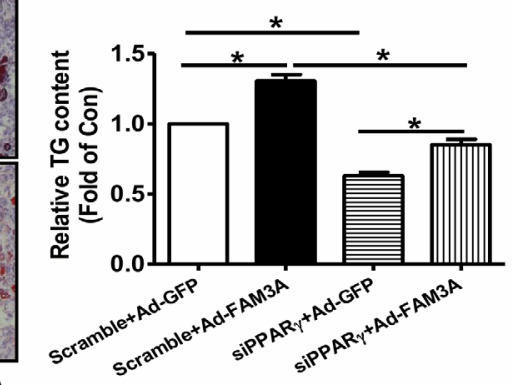

C

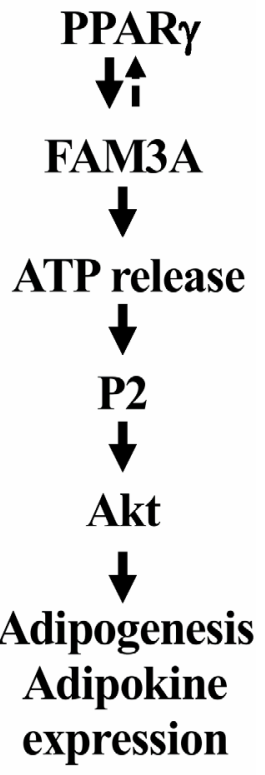

Figure 8: Knockdown of PPAR $\gamma$ failed to affect FAM3A-induced Akt activation in 3T3-L1 preadipocytes. 3T3-L1 preadipocytes were transfected with siPPAR $\gamma(50 \mathrm{nM})$ or scrambled siRNA $(50 \mathrm{nM})$ for 24 hours, and infected with Ad-GFP or AdFAM3A for 24 hours (Day 0). The infected cells were then induced for differentiation as above. (A) PPAR $\gamma$ silencing failed to affect FAM3A-induced Akt phosphorylation at Day 0. Representative gel images were shown in left panel, and quantitative data in right panel. $\mathrm{N}=4,{ }^{*} \mathrm{p}<0.05$ between indicated groups. (B) FAM3A promoted the adipogenesis of 3T3-L1 cells after PPAR $\gamma$ knockdown. Representative images of differentiated 3T3-L1 cells with Oil Red O staining were shown in left panel, and quantitative analyses of TG levels in right panel. $\mathrm{N}=3,{ }^{*} \mathrm{p}<0.05$ between indicated groups. (C) Schematic summary of the adipogenic effect of FAM3A in preadipocyte differentiation. FAM3A promotes the adipogenesis of preadipocytes by activating ATP-P2 receptor-Akt pathway. 
cells, indicating that ATP-P2 receptor-Akt pathway plays a decisive role in FAM3A-induced preadipocyte adipogenesis. In support, it had been previously reported that addition of exogenous ATP alone fails to stimulate adipogenesis, but it augments the adipogenic effects of dexamethasone and 3-isobutyl-1-methylxanthine in 3T3L1 preadipocytes [23].

Regarding the role of FAM3A regulating adipogenic process, several issues should also be noted. FAM3A silencing inhibited the adipogenesis and adipokine expression/secretion in 3T3-L1 preadipocytes. However, although FAM3A overexpression enhanced the adipogenesis of 3T3-L1 preadipocytes, it failed to further increase adipokine expression/secretion. It is possible that adipokine expression are already at high levels during the adipogenesis, thus FAM3A overexpression fails to further elevate their levels in 3T3-L1 cells. Mice with adiposespecific deletion of PPAR $\gamma$ exhibit lipodystrophy and dramatically decreased serum adiponectin and resistin levels when compared with wild type mice [7]. In contrast, although mice with adipose-specific overexpression of PPAR $\gamma$ exhibit increased adipose mass, the serum adiponectin and resistin levels remained unchanged when compared with wild type mice [6]. Although FAM3A is a target gene of PPAR $\gamma$, overexpression or silencing of FAM3A increases or decreases PPAR $\gamma 2$ protein level during adipogenesis. It had been reported that Akt activation upregulated PPAR $\gamma$ expression and activity by inactivating FOXO1 or activating mTOR during the adipogenesis of 3T3-L1 cells [31-34]. Our result further indicated that FAM3A-induced Akt activation is not dependent on PPAR $\gamma$ at the beginning of 3T3-L1 differentiation. So, FAM3A-mediated increase in PPAR $\gamma 2$ expression is likely due to long term Akt activation. It is also possible that an increase in PPAR $\gamma 2$ protein level is the consequence of 3T3-L1 preadipocyte maturation in case of FAM3A overexpression. Overall, there is a crosstalk between FAM $3 \mathrm{~A}$ and PPAR $\gamma 2$ expression during preadipocyte differentiation. It had been previously reported that in adipose tissue of fasting mice, PPAR $\gamma 2$ protein was reduced, whereas PPAR $\gamma 1$ protein remained unchanged when compared with that of fed mice [35]. Mice with PPAR $\gamma 2$ deletion exhibit reduced white adipose tissues when compared with wild type mice [36]. These findings suggested that PPAR $\gamma 2$ plays a crucial role in the regulation of preadipocyte adipogenesis. It should also be noted that FAM3A expression may also be regulated by other factors such as nutrients, other transcription factors or miRNAs beyond PPAR $\gamma$ during preadipocytes adipogenesis. Further study are still needed to clarify this crosstalk and its precise role in preadipocyte adipogenesis using mice with specific deletion of FAM3A and/or PPAR $\gamma$ in white adipose tissue in the future.

In summary, FAM3A plays important roles in regulating preadipocyte adipogenesis via the enhancement of ATP production, which activates Akt proliferative pathway(s) through P2 receptors. Under obese condition, a decrease in FAM3A expression in adipose tissue could contribute to the development of adipose dysfunction and type 2 diabetes (Figure 8C).

\section{MATERIALS AND METHODS}

\section{Experimental animals}

Eight-week-old male C57BL/6 mice and 8 to 12-week-old male $\mathrm{db} / \mathrm{db}$ and $\mathrm{db} / \mathrm{m}$ mice on a C57BSK background (Jackson Laboratory, USA) were used in this study. $d b / d b$ and $d b / m$ mice were age matched. C57BL/6 mice were fed with a $45 \%$ high fat diet (HFD) or normal diet (ND) for 12 weeks [20]. Normal or HFD-fed C57BL/6 mice were daily administrated with rosiglitazone (AVANDIA, $30 \mathrm{mg} / \mathrm{kg} /$ day) for 30 days (Control mice were administrated with saline). Adipose tissues in this study are referred to be the epididymal white adipose tissues. All procedures involving experimental animals were approved by the Institutional Animal Care and Use Committee of Peking University Health Science Center that complies with the Guide for the Care and Use of Laboratory Animals published by the US National Institutes of Health (NIH Publication No. 85-23, revised 1996).

\section{Cell culture and differentiation}

Mouse 3T3-L1 preadipocytes were cultured in DMEM supplemented with $10 \%$ FBS and penicillinstreptomycin at $37^{\circ} \mathrm{C}$ in $5 \% \mathrm{CO}_{2}$. Two days post confluence (designed as Day 0), the cells were stimulated with MDI $(0.5 \mathrm{mM}$ IBMX, $1 \mu \mathrm{M}$ dexamethasone and $10 \mu \mathrm{g} / \mathrm{mL}$ insulin) induction media. In rosiglitazone group, $10 \mu \mathrm{M}$ rosiglitazone (Sigma) was added with MDI in Day 0. Two days after MDI (Day 2) change the media to Insulin Media $(10 \mu \mathrm{g} / \mathrm{mL}$ insulin). Two days later (Day 4) change media to 10\% FBS/DMEM. Feed cells with $10 \%$ FBS/DMEM every two days. 3T3-L1 cells were collected on Day 0, Day 4 and Day 8 for further analyses, respectively.

\section{Overexpression or knockdown of FAM3A in 3T3-L1 preadipocytes}

3T3-L1 preadipocytes were infected with 50 MOI of Ad-GFP or Ad-FAM3A for 24 hours before induction of differentiation (Day -1). PPADS $(50 \mu \mathrm{M})$ was added to media from Day 0 to Day 8 every 24 hours. 3T3-L1 cells were collected on Day 0, Day 4 and Day 8 for further analyses, respectively. To knockdown FAM3A gene expression, 3T3-L1 preadipocytes were transfected with $50 \mathrm{~nm}$ siFAM3A mixture against FAM3A cDNA coding sequence synthesized by Beijing Biolino Co., Ltd. (siRNA sequences were listed in 
Supplementary Table 1. Scrambled siRNA sequences from the same company were used as control) for 24 hours before induction of differentiation (Day -1). Cells were collected on Day 0, Day 4 and Day 8 for analysis, respectively.

\section{Oil Red O staining}

At Day 0, Day 4 and Day 8 after induction, 3T3L1 cells were fixed with $4 \%$ formaldehyde for $30 \mathrm{~min}$ and then subjected to Oil Red O staining for $1 \mathrm{~h}$ to visualize neutral lipid deposition in the cells. Relative intracellular TG content was quantified by eluting Oil Red $\mathrm{O}$ staining with $100 \%$ isopropyl alcohol and measured at $570 \mathrm{~nm}$ with a microplate reader.

\section{Quantitative PCR assays}

Total RNA of cells and tissues were isolated using TRIzol reagent (Invitrogen). The complementary DNA was synthesized using RevertAid First Strand cDNA Synthesis Kit (Thermo). Quantitative PCR was performed with the Biorad System using SRBR Green 1 (TOYOBO) as a fluorescent probe. Target gene mRNA level was normalized to that of $\beta$-actin in the same sample as described previously using $2^{-\Delta \Delta C t}$ methods $[19,20]$. All of the primer sequences used in this study are listed in Supplementary Table 2.

\section{Western blot analyses}

Adipose tissues and cells were lysed in Roth lysis buffer. $60 \mu \mathrm{g}$ cell protein and $80 \mu \mathrm{g}$ adipose tissue protein were separated by $12 \%$ SDS-PAGE, and then transferred to the NC (nitrocellulose filter) membrane. The membrane was incubated in 1:500-1:1000 primary antibody. Primary antibodies were: anti-PPAR $\gamma$ antibodies (Santa Cruz Biotechnology, sc-7196), antiFAM3A antibodies (Sigma), anti-pAkt (Ser473) and anti-Akt antibodies (Cell Signaling Technology). After incubated with the second antibody, the membrane was exposed to ECL. After immunoblotting assays, the membrane was stripped with $0.2 \mathrm{~N} \mathrm{NaOH}$ and re-probed for $\beta$-actin as loading controls.

\section{ELISA}

Cell culture medium of 3T3-L1 cells on differentiation Day 0, Day 4 and Day 8 were collected and centrifuged at $12000 \mathrm{rpm}$ at $4^{\circ} \mathrm{C}$ for 5 minutes, and then the supernatant were maintained for determination of adipokine levels. The levels of adiponectin and resistin were determined using ELISA Kit (Cloud-Clone Corp. SEA605Mu and SEA847Mu) according to the manufacturer's instruction.

\section{Mitochondria isolation}

Mitochondria were isolated from adipose tissue or 3T3-L1 cells using the Mitochondria/Cytosol Fractionation Kit (Pierce). In brief, 3T3-L1 cells or adipose tissue were homogenized in Mito-Cyto extraction buffer, and then the lysate was centrifuged twice at $800 \mathrm{~g}$ for 5 minutes to pellet the nucleus and cell debris. The supernatant was collected and centrifuged at $10,000 \mathrm{~g}$ for 10 min to pellet mitochondria, and then the mitochondria were washed at least three times with lysis buffer to avoid cytoplasmic proteins contamination.

\section{ATP content determination}

ATP content in the cells and medium was determined using ATP-Lite Assay Kit (Vigorous Biotechnology Beijing Co., Ltd) as detailed previously [20]. For determination of relative ATP level in the cells or tissues, the ATP content values (nmol) were first normalized to the protein content (nmol/mg.protein) in the same sample, and then normalized to the control values. For determination of relative ATP level in the medium, the absolute concentration was determined and normalized to the control value.

\section{siRNA knockdown of PPAR $\gamma$ in 3T3-L1 preadipocytes}

3T3-L1 preadipocytes were transfected with siRNA against both PPAR $\gamma 1$ and PPAR $\gamma 2(50 \mathrm{nM})$ or scrambled siRNA (50 nM) mixture using VigoFect (Vigorous Biotechnology, Beijing, China) for 24 hours, and then infected with Ad-GFP or Ad-FAM3A for 24 hours (Day 0). The infected cells were further induced for differentiation as above. The siRNA sequences were provided in Supplementary Table 1.

\section{Statistical analysis}

Data are presented as mean \pm SEM. Statistical significance of differences between groups was analyzed by unpaired $t$ test. Statistical significance was set at $\mathrm{P}<0.05$.

\section{Abbreviations}

aP2: adipocyte fatty acid-binding protein; $\mathrm{C} / \mathrm{EBP} \alpha$ : CCAAT-enhancer-binding protein $\alpha$; C/EBP $\delta$ : CCAATenhancer-binding protein $\delta$; EWAT: epididymal white adipose tissue; FAM3A: family with sequence similarity 3 member A; HFD: high fat diet; IBMX: 3-isobutyl-1methylxanthine; MOI: multiplicity of infection; NAFLD: non-alcoholic fatty liver disease; PANDER: pancreatic derived factor; PI3K: phosphatidylinositol 3 kinase; PPAR $\gamma$ : peroxisome proliferator-activated receptor $\gamma$; siRNA: small interference RNA; TG: triglycerides; UCP-2: uncoupling protein 2; VSMCs: vascular smooth muscular cells. 


\section{Author contributions}

This work was conceived and designed by J.Y. and Y.L. Y.C. and J.L together performed the main experiments and wrote the manuscript. Z.C. performed mitochondria extraction and ATP content determination. N.L., W.P., X.P. and M.L. assisted experimental process and animal experiments. W.Y. and X.H. gave technical suggestion during the research. B.G. and Q.C. assisted the experimental process and provided necessary comments. Y.C. and J.L. wrote the manuscript. J.Y. and Y.L. reviewed and revised the manuscript. All authors reviewed the manuscript.

\section{CONFLICTS OF INTEREST}

The authors declare that there is no conflicts of interest.

\section{FUNDING}

This study was supported by grants from by the Ministry of Science and Technology (2016YFC1304800) and the Natural Science Foundation of China (81670787/8 $1200625 / 81200626 / 81471035 / 81670748 / 81322011)$. This work also supported by Specialized Research Fund for the Doctoral Program of Higher Education (20120001120086 /20120001120057).

\section{REFERENCES}

1. Chan JM, Rimm EB, Colditz GA, Stampfer MJ, Willett WC. Obesity, fat distribution, and weight gain as risk factors for clinical diabetes in men. Diabetes Care. 1994; 17:961-969.

2. Colditz GA, Willett WC, Rotnitzky A, Manson JE. Weight gain as a risk factor for clinical diabetes mellitus in women. Ann Intern Med. 1995; 122:481-486.

3. Lefterova MI, Haakonsson AK, Lazar MA, Mandrup S. PPAR $\gamma$ and the global map of adipogenesis and beyond. Trends Endocrinol Metab. 2014; 25:293-302.

4. Sauer S. Ligands for the nuclear peroxisome proliferatoractivated receptor gamma. Trends Pharmacol Sci. 2015; 36:688-704.

5. Wright MB, Bortolini M, Tadayyon M, Bopst $M$. Minireview: challenges and opportunities in development of PPAR agonists. Mol Endocrinol. 2014; 28:1756-1768.

6. Sugii S, Olson P, Sears DD, Saberi M, Atkins AR, Barish GD, Hong SH, Castro GL, Yin YQ, Nelson MC, Hsiao G, Greaves DR, Downes M, et al. PPAR $\gamma$ activation in adipocytes is sufficient for systemic insulin sensitization. Proc Natl Acad Sci U S A. 2009; 106:22504-22509.

7. Wang F, Mullican SE, DiSpirito JR, Peed LC, Lazar MA. Lipoatrophy and severe metabolic disturbance in mice with fat-specific deletion of PPARgamma. Proc Natl Acad Sci U S A. 2013; 110:18656-18661.

8. Gustafson B, Hedjazifar S, Gogg S, Hammarstedt A, Smith U. Insulin resistance and impaired adipogenesis. Trends Endocrinol Metab. 2015; 26:193-200.

9. Dawes TD, Turincio R, Jones SW, Rodriguez RA, Gadiagellan D, Thana P, Clark KR, Gustafson AE, Orren L, Liimatta M, Gross DP, Maurer T, Beresini MH. Compound transfer by acoustic droplet ejection promotes quality and efficiency in ultra-high-throughput screening campaigns. J Lab Autom. 2016; 21:64-75.

10. Payne VA, Au WS, Lowe CE, Rahman SM, Friedman JE, O'Rahilly S, Rochford JJ. C/EBP transcription factors regulate SREBP1c gene expression during adipogenesis. Biochem J. 2010; 425:215-223.

11. Bays H. Adiposopathy, "sick fat," Ockham's razor, and resolution of the obesity paradox. Curr Atheroscler Rep. 2014; 16:409.

12. Lopategi A, Lopez-Vicario C, Alcaraz-Quiles J, GarciaAlonso V, Rius B, Titos E, Claria J. Role of bioactive lipid mediators in obese adipose tissue inflammation and endocrine dysfunction. Mol Cell Endocrinol. 2016; 419:44-59.

13. Wang C, Guan Y, Yang J. Cytokines in the progression of pancreatic beta-cell dysfunction. Int J Endocrinol. 2010; 2010:515136.

14. Yang J, Kang J, Guan Y. The mechanisms linking adiposopathy to type 2 diabetes. Front Med. 2013; 7:433-444.

15. Jung CH, Kang YM, Jang JE, Hwang JY, Kim EH, Park JY, Kim HK, Lee WJ. Fatty liver index is a risk determinant of incident type 2 diabetes in a metabolically healthy population with obesity. Obesity. 2016; 24:1373-1379.

16. Samocha-Bonet D, Dixit VD, Kahn CR, Leibel RL, Lin X, Nieuwdorp M, Pietilainen KH, Rabasa-Lhoret R, Roden M, Scherer PE, Klein S, Ravussin E. Metabolically healthy and unhealthy obese - the 2013 Stock Conference report. Obes Rev. 2014; 15:697-708.

17. Bays H. Central obesity as a clinical marker of adiposopathy; increased visceral adiposity as a surrogate marker for global fat dysfunction. Curr Opin Endocrinol Diabetes Obes. 2014; 21:345-351.

18. Moreno-Indias I, Tinahones FJ. Impaired adipose tissue expandability and lipogenic capacities as ones of the main causes of metabolic disorders. J Diabetes Res. 2015; 2015:970375.

19. Zhou Y, Jia S, Wang C, Chen Z, Chi Y, Li J, Xu G, Guan Y, Yang J. FAM3A is a target gene of peroxisome proliferatoractivated receptor gamma. Biochim Biophys Acta. 2013; 1830:4160-4170.

20. Wang C, Chi Y, Li J, Miao Y, Li S, Su W, Jia S, Chen Z, Du S, Zhang X, Zhou Y, Wu W, Zhu M, et al. FAM3A activates PI3K p110alpha/Akt signaling to ameliorate hepatic gluconeogenesis and lipogenesis. Hepatology. 2014; 59:1779-1790. 
21. Jia S, Chen Z, Li J, Chi Y, Wang J, Li S, Luo Y, Geng B, Wang C, Cui Q, Guan Y, Yang J. FAM3A promotes vascular smooth muscle cell proliferation and migration and exacerbates neointima formation in rat artery after balloon injury. J Mol Cell Cardiol. 2014; 74:173-182.

22. Newton BW, Cologna SM, Moya C, Russell DH, Russell WK, Jayaraman A. Proteomic analysis of 3T3-L1 adipocyte mitochondria during differentiation and enlargement. J Proteome Res. 2011; 10:4692-4702.

23. Omatsu-Kanbe $\mathrm{M}$, Inoue $\mathrm{K}$, Fujii $\mathrm{Y}$, Yamamoto $\mathrm{T}$, Isono T, Fujita N, Matsuura H. Effect of ATP on preadipocyte migration and adipocyte differentiation by activating P2Y receptors in 3T3-L1 cells. Biochem J. 2006; 393:171-180.

24. Chen YC, Wu YT, Wei YH. Depletion of mitoferrins leads to mitochondrial dysfunction and impairment of adipogenic differentiation in 3T3-L1 preadipocytes. Free Radic Res. 2015; 49:1285-1295.

25. Sun $\mathrm{X}$, Zemel MB. Role of uncoupling protein 2 (UCP2) expression and 1alpha, 25-dihydroxyvitamin D3 in modulating adipocyte apoptosis. FASEB J. 2004; 18:1430-1432.

26. Rong JX, Qiu Y, Hansen MK, Zhu L, Zhang V, Xie M, Okamoto Y, Mattie MD, Higashiyama H, Asano S, Strum JC, Ryan TE. Adipose mitochondrial biogenesis is suppressed in $\mathrm{db} / \mathrm{db}$ and high-fat diet-fed mice and improved by rosiglitazone. Diabetes. 2007; 56:1751-1760.

27. Wang S, Xu Q, Shu G, Wang L, Gao P, Xi Q, Zhang Y, Jiang Q, Zhu X. N-Oleoyl glycine, a lipoamino acid, stimulates adipogenesis associated with activation of $\mathrm{CB} 1$ receptor and Akt signaling pathway in 3T3-L1 adipocyte. Biochem Biophys Res Commun. 2015; 466:438-443.

28. Li M, Liu Z, Zhang Z, Liu G, Sun S, Sun C. miR-103 promotes 3T3-L1 cell adipogenesis through AKT/mTOR signal pathway with its target being MEF2D. Biol Chem. 2015; 396:235-244.

29. Simon BR, Parlee SD, Learman BS, Mori H, Scheller EL, Cawthorn WP, Ning X, Gallagher K, Tyrberg B,
Assadi-Porter FM, Evans CR, MacDougald OA. Artificial sweeteners stimulate adipogenesis and suppress lipolysis independently of sweet taste receptors. J Biol Chem. 2013; 288:32475-32489.

30. Kwon JY, Seo SG, Heo YS, Yue S, Cheng JX, Lee KW, Kim KH. Piceatannol, natural polyphenolic stilbene, inhibits adipogenesis via modulation of mitotic clonal expansion and insulin receptor-dependent insulin signaling in early phase of differentiation. J Biol Chem. 2012; 287:11566-11578.

31. Baba S, Ueno Y, Kikuchi T, Tanaka R, Fujimori K. A limonoid kihadanin $\mathrm{b}$ from immature citrus unshiu peels suppresses adipogenesis through repression of the AktFOXO1-PPAR $\gamma$ axis in adipocytes. J Agric Food Chem. 2016; 64:9607-9615.

32. Wu M, Liu D, Zeng R, Xian T, Lu Y, Zeng G, Sun Z, Huang B, Huang Q. Epigallocatechin-3-gallate inhibits adipogenesis through down-regulation of PPAR $\gamma$ and FAS expression mediated by PI3K-AKT signaling in 3T3-L1 cells. Eur J Pharmacol. 2016; 795:134-142.

33. Kim JE, Chen J. Regulation of peroxisome proliferatoractivated receptor-gamma activity by mammalian target of rapamycin and amino acids in adipogenesis. Diabetes. 2004; 53:2748-2756.

34. Soumya SJ, Binu S, Helen A, Reddanna P, Sudhakaran PR. 15(S)-HETE-induced angiogenesis in adipose tissue is mediated through activation of PI3K/Akt/mTOR signaling pathway. Biochem Cell Biol. 2013; 91:498-505.

35. Vidal-Puig A, Jimenez-Linan M, Lowell BB, Hamann A, $\mathrm{Hu}$ E, Spiegelman B, Flier JS, Moller DE. Regulation of PPAR gamma gene expression by nutrition and obesity in rodents. J Clin Invest. 1996; 97:2553-2561.

36. Zhang J, Fu M, Cui T, Xiong C, Xu K, Zhong W, Xiao Y, Floyd D, Liang J, Li E, Song Q, Chen YE. Selective disruption of PPARgamma 2 impairs the development of adipose tissue and insulin sensitivity. Proc Natl Acad Sci U S A. 2004; 101:10703-10708. 